\title{
Comparative transcriptome sequencing of germline and somatic tissues of the Ascaris suum gonad
}

Xuan Ma', Yingjie Zhu' ${ }^{2}$, Chunfang $\mathrm{Li}^{2}$, Yunlong Shang ${ }^{1}$, Fanjing Meng ${ }^{3}$, Shilin Chen ${ }^{2}$ and Long Miao ${ }^{*}$

\begin{abstract}
Background: Ascaris suum (large roundworm of pigs) is a parasitic nematode that causes substantial losses to the meat industry. This nematode is suitable for biochemical studies because, unlike C. elegans, homogeneous tissue samples can be obtained by dissection. It has large sperm, produced in great numbers that permit biochemical studies of sperm motility. Widespread study of A. suum would be facilitated by more comprehensive genome resources and, to this end, we have produced a gonad transcriptome of $A$. suum.

Results: Two 454 pyrosequencing runs generated 572,982 and 588,651 reads for germline (TES) and somatic (VAS) tissues of the A. suum gonad, respectively. $86 \%$ of the high-quality $(\mathrm{HQ})$ reads were assembled into 9,955 contigs and 69,791 HQ reads remained as singletons. 2.4 million bp of unique sequences were obtained with a coverage that reached 16.1 -fold. 4,877 contigs and 14,339 singletons were annotated according to the C. elegans protein and the Kyoto Encyclopedia of Genes and Genomes (KEGG) protein databases. Comparison of TES and VAS transcriptomes demonstrated that genes participating in DNA replication, RNA transcription and ubiquitinproteasome pathways are expressed at significantly higher levels in TES tissues than in VAS tissues. Comparison of the A. suum TES transcriptome with the C. elegans microarray dataset identified 165 A. suum germline-enriched genes (83\% are spermatogenesis-enriched). Many of these genes encode serine/threonine kinases and phosphatases (KPs) as well as tyrosine KPs. Immunoblot analysis further suggested a critical role of phosphorylation in both testis development and spermatogenesis. A total of 2,681 A. suum genes were identified to have associated RNAi phenotypes in C. elegans, the majority of which display embryonic lethality, slow growth, larval arrest or sterility.

Conclusions: Using deep sequencing technology, this study has produced a gonad transcriptome of A. suum. By comparison with C. elegans datasets, we identified sets of genes associated with spermatogenesis and gonad development in A. suum. The newly identified genes encoding KPs may help determine signaling pathways that operate during spermatogenesis. A large portion of A. suum gonadal genes have related RNAi phenotypes in $C$. elegans and, thus, might be RNAi targets for parasite control.
\end{abstract}

\section{Background}

The genus Ascaris, also known as the "giant intestinal roundworms", contains the largest intestinal nematode species. Ascaris lumbricoides causes the commonest helminth infection of humans, whereas a closely related species, Ascaris suum, typically infects pigs and causes substantial financial losses to the meat industry. The $A$.

\footnotetext{
* Correspondence: Imiao@moon.ibp.ac.cn

'Noncoding RNA laboratory, National Laboratory of Biomacromolecules, Institute of Biophysics, Chinese Academy of Sciences, Beijing 100101, China Full list of author information is available at the end of the article
}

suum female is capable of producing more than 200,000 eggs per day and these eggs can survive and remain infective after many years in soil [1]. At present, there is no effective alternative to chemical control of intestinal parasites and resistance to anthelmintics has become an emerging problem [2]. Greater knowledge of nematode biology is urgently needed to enable the development of new biotechnological tools (e.g., RNA interference) for parasite control.

To better understand the molecular and biochemical basis of nematode development, nematode EST projects
C Biomed Central

ㄷ 2011 Ma et al; licensee BioMed Central Ltd. This is an Open Access article distributed under the terms of the Creative Commons Attribution License (http://creativecommons.org/licenses/by/2.0), which permits unrestricted use, distribution, and reproduction in any medium, provided the original work is properly cited. 
have generated more than 250,000 ESTs from 30 species, including $A$. suum [3]. Large-scale EST datasets have also been acquired by next-generation sequencing (NGS) technologies and the associated bioinformatic pipeline has been developed [4,5]. This vast collection of ESTs combined with the extensive knowledge of Caenorhabditis elegans biology provides opportunities to elucidate functionally conserved mechanisms in nematode biology. Employment of NGS technologies has greatly accelerated the 959 Nematode Genomes project http://www.nematodes.org/nematodegenomes/index. php/Main_Page. Genome sequencing of A. suum somatic cells is ongoing http://www.sanger.ac.uk/ resources/downloads/helminths/ascaris-suum.html, and a draft genome and transcriptome of $A$. suum is now available http://www.nematode.net/NN3_frontpage.cgi? navbar_selection=home\&subnav_selection=asuum_ftp. To date, there have been 38 A. suum EST libraries with $\sim 55,000$ sequences available in the NEMBASE4 database http://www.nematodes.org/nembase4/ and these ESTs were obtained using conventional cDNA library sequencing technology.

In the present study, we applied 454 pyrosequencing technology to unravel the transcriptome of the male $A$. suum gonad, the organ for reproduction. A. suum males have a large gonad that can be readily isolated by dissection to provide large numbers of sperm that are suitable for biochemical and cell biological studies [6]. The male A. suum gonad is composed of three distinct regions; the testis and seminal vesicle form germline tissue and the glandular vas deferens forms somatic tissue. Sperm are stored in the seminal vesicle. During copulation, the spherical, non-motile sperm are activated into bipolar, amoeboid spermatozoa by an unknown component secreted by the glandular vas deferens. The motility of amoeboid sperm is driven by the regulated assembly and disassembly of major sperm protein (MSP) cytoskeleton $[7,8]$. The mechanism of sperm activation is poorly understood and the details of MSP-based sperm motility are yet to be determined, although several proteins (e.g., MPOP, MFPs and PP2A) that participate in the dynamics of the MSP cytoskeleton have been identified [9-12]. Despite the advantages of large gametes and the easy isolation of reproductive fluids from $A$. suum, there have been few studies focusing on sperm chromatin or on distinctions between germline and somatic tissues in A. suum. In addition, chromatin diminution in A. suum represents a fascinating exception to the general rule of the constancy of the genome. However, the complex mechanism of this phenomenon, involving DNA degradation and new telomere addition remain an enigma [13-18]. One of the barriers to answering the above questions is the lack of gene expression data for the reproductive tissues of $A$. suum.
To facilitate diverse studies concerning reproductive biology in $A$. suum, we acquired the transcriptomes of germline and somatic tissues of $A$. suum gonad using the RNA-seq approach. Comparison of these two tissues showed that the nucleic acid metabolic and proteasomeubiquitin pathways are more active in the germline than in the soma. Further comparison with C. elegans microarray data identified 165 conserved germline-enriched genes in A. suum. We also categorized the RNAi phenotypes for A. suum gonadal genes, taking advantage of the C. elegans RNAi phenotype database. Therefore, these $A$. suum transcriptome data provide a valuable platform for both fundamental biological studies (e.g., MSP-based sperm motility and spermatogenesis studies) and for research concerning parasite control (e.g. use of RNAi).

\section{Results}

454 sequencing and de novo assembly of $A$. suum gonad transcriptome

The male $A$. suum gonad was dissected into two parts: testis and seminal vesicle (TES) and glandular vas deferens (VAS), and both samples were subjected to total RNA extraction followed by cDNA synthesis. Secondstrand cDNAs with trimmed poly(A) tails were used for high-throughput sequencing on a 454 GS FLX Titanium platform. We performed two runs that produced $\sim 1.16$ million raw reads constituting a total of $\sim 0.4$ billion base-pairs (bp). The majority of the reads were over 400 $\mathrm{bp}$ and the average length of the reads was $\sim 356 \mathrm{bp}$. The size distribution of the raw reads from both samples is shown in Figure 1A. To acquire high-quality reads, we filtered out the reads shorter than $50 \mathrm{bp}$, which account for $4.3 \%$ of total reads. These high-quality reads were then used in de novo assembly using Newbler (Version 2.3). 999,214 reads from either TES or VAS were assembled into 9,955 contigs. These contigs range from $100 \mathrm{bp}$ to $6,649 \mathrm{bp}$ and $97.6 \%$ of them were assembled from three or more reads. The size distribution of the contigs is shown in Figure 1B. The number of singletons in TES and VAS datasets was 30,137 and 39,654 , respectively, and they together comprised $10 \%$ of total reads. We obtained 2.4 Mbp of unique sequences with a coverage that reached 16.1-fold. All unique sequences are available at http://159.226.118.206/miaolab/index.htm. The contigs longer than 200 bp have been deposited in the GenBank Transcriptome Shotgun Assembly (TSA) database under the accession numbers JO467643-JO475858. A summary of 454 sequencing and assembly is shown in Table 1.

The current $A$. suum testis EST library in NEMBASE4 has collected 2,868 ESTs. These ESTs correspond to 595 homologous genes in $C$. elegans (BLAST cutoff E-value $\left.=1 \mathrm{e}^{-5}\right)$. In contrast, our A. suum gonad transcriptome 


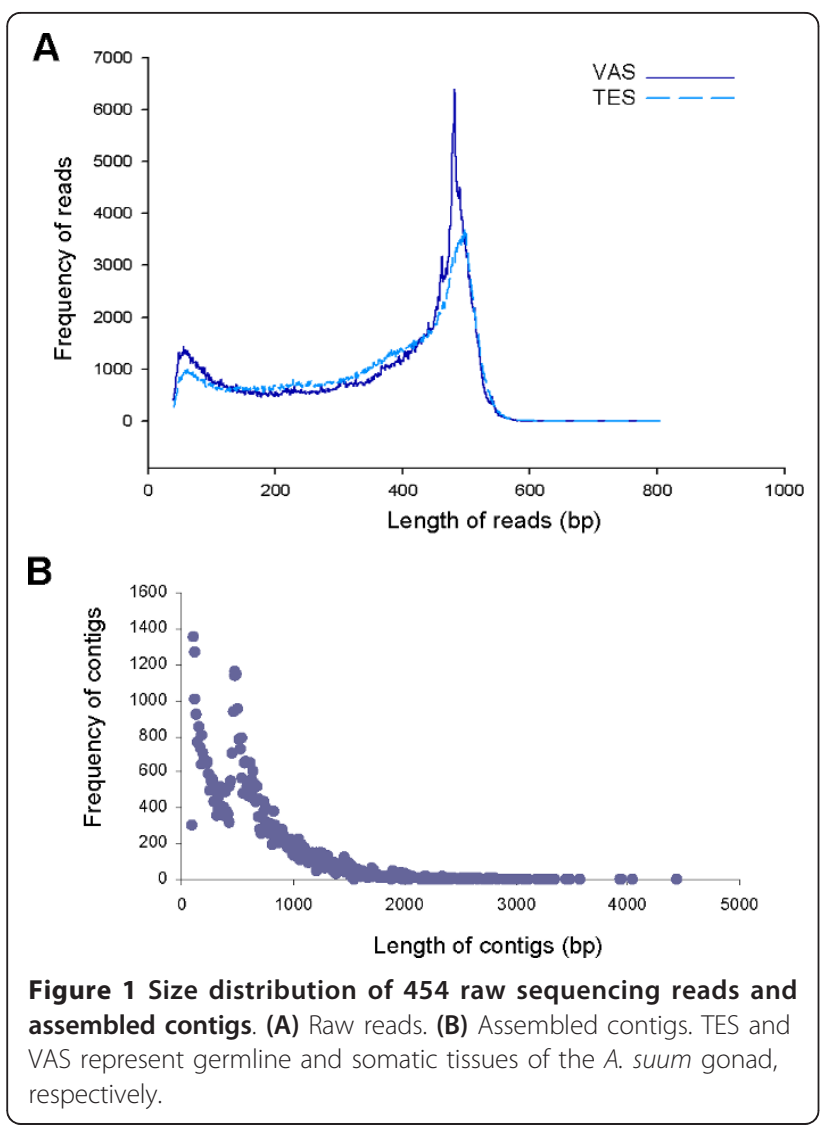

corresponds to 4,207 homologous genes in C. elegans and 3,686 novel gonadal genes were identified (Additional file 1). This suggests our A. suum gonad transcriptome has a deeper coverage than the conventional EST library.

Functional assignments of $A$. suum 454 sequencing data To annotate the $A$. suum 454 transcriptome data, we compared all unique sequences against the C. elegans protein database in WormBase, as well as against the

Table 1 Summary of 454 sequencing and assembly

\begin{tabular}{lll}
\hline & TES* & VAS* \\
\hline Sequencing & & \\
No. of reads & 572,982 & 588,651 \\
No. of base-pairs & $204,129,803$ & $209,849,741$ \\
Average read length (bp) & 356.3 & 356.5 \\
\hline Assembly & & \\
No. of singletons (> 50 bp) & 30,137 & 39,654 \\
Average length of singletons & 287.6 & 251.5 \\
No. of contigs & 9,955 & \\
Average length of contigs (bp) & 621.8 & \\
\hline Total coverage (bp) & $24,828,337$ &
\end{tabular}

*Note: TES and VAS represent germline and somatic tissues of $A$. suum gonad, respectively.
Kyoto Encyclopedia of Genes and Genomes (KEGG) protein databases using BLASTX (cutoff E-value $=1 \mathrm{e}^{-5}$ ). A total of 4,877 contigs (49\%) and 14,339 singletons (20.5\%) were annotated. A large portion of the 454 sequences have not been functionally defined. Some sequences can be annotated by increasing the E-value and others may represent $A$. suum specific genes. In summary, 9,822 unique sequences (corresponding to 5,683 gene models) were annotated in the TES dataset and 12,123 unique sequences (corresponding to 4,122 gene models) were annotated in the VAS dataset (Additional file 2). Although $\sim 2,000$ more sequences were assigned in the VAS dataset compared with the TES dataset, TES has $\sim 1,500$ more gene models than VAS suggesting that there are more diverse genes expressed in TES than in VAS tissues.

Tubulin genes ( $>12,000$ reads) are the most abundant transcripts in the A. suum gonad; the fibulin genes (> 10,000 reads), whose activity is essential for gonad and body morphology in C. elegans [19], are also highly abundant. The expression of genes encoding intermediate filament proteins, heat shock proteins, ribosomal proteins, aldehyde reductase and major sperm proteins were also enriched. It should be noted that among the 100 most highly enriched genes, over half have not been functionally characterized.

\section{Metabolic pathway mapping}

To gain insight into A. suum gonad metabolic pathways, we mapped the $C$. elegans homologues of $A$. suum genes to the KEGG pathways. A total of 5,426 unique $A$. suum sequences corresponding to 850 homologous genes in C. elegans were assigned to metabolic pathways (Additional file 3). Among the 5,426 sequences, 33.1\% were expressed in TES and $77.9 \%$ were expressed in VAS; only $11.1 \%$ were expressed in both TES and VAS. This suggested TES and VAS express distinct groups of genes that participate in their respective metabolic processes. As shown in Figure 2, the majority of TES and VAS genes are classified into pathways for transcription, transport and catabolism, folding, sorting and degradation, translation, carbohydrate and amino acid metabolism. In VAS, a large number of genes participate in the transport and catabolism pathway (highlighted by blue circle), while TES has twice the number of genes involved in the transcription pathway and the folding, sorting and degradation pathway as VAS (highlighted by red circles).

\section{Comparative analysis of TES and VAS datasets}

Annotation of the TES and VAS datasets has indicated differences in gene expression in a tissue-specific manner. The majority of TES tissues comprise germ cells while VAS tissues are exclusively composed of somatic 


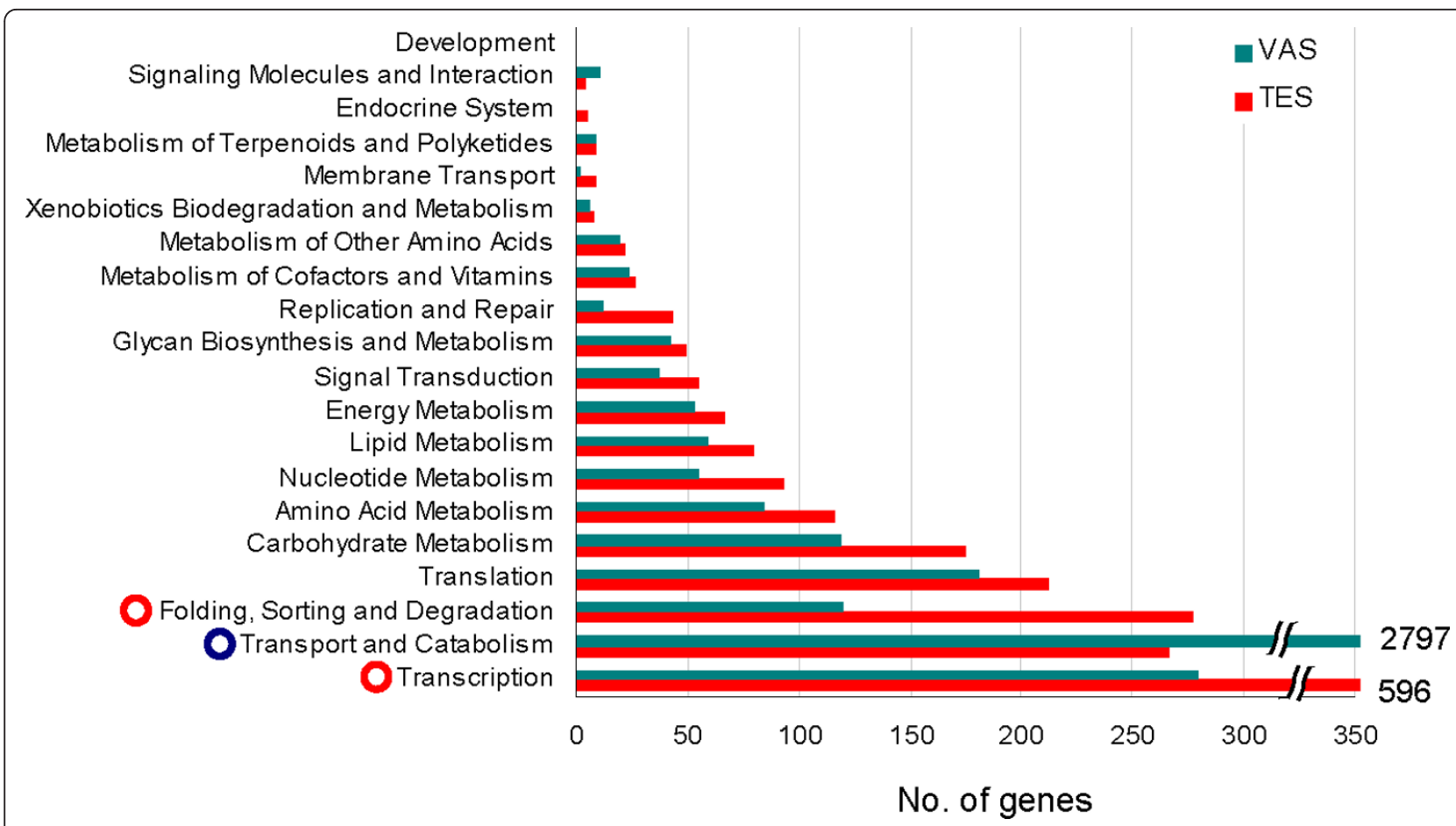

Figure 2 Distribution of the number of genes expressed in various metabolic pathways. Red and green bars represent the TES and VAS datasets, respectively. Different metabolic pathways are shown on the left. Blue circle marks the metabolic process requiring the most number of genes in VAS tissues; red circles mark the processes that require twice as many genes in TES compared with VAS tissues.

cells. To further characterize their differential geneexpression patterns, we used the contig dataset to compare their transcriptomes according to the number of reads, and a global expression profiling for all contigs is shown in Figure 3A. These two tissues have dramatically different expression patterns. To quantify these differences, we normalized the expression levels of TES and VAS before calculating the reads-ratio of TES to VAS. The thresholds 10 and 0.1 were set to identify the highly expressed contigs in TES and VAS, respectively. This analysis identified 3,110 contigs having levels of expression that were at least 10 -fold higher in TES compared with VAS, and 1,165 contigs whose levels of expression were at least 10-fold higher in VAS than in TES (Additional file 4).

To highlight the functions of differentially expressed genes between TES and VAS, the contigs having 10-fold higher levels of expression were searched against the STRING database http://string-db.org/ to identify the functional associations of these genes. The result demonstrated that, compared with the somatic VAS tissues, the germline TES tissues has a more complex gene/protein interaction network (Additional file 5). In the germline, the genes encoding proteins involved in DNA replication and RNA transcription are highly enriched; the germline also expresses a large number of genes participating in the proteasome and ubiquitin- mediated proteolysis pathways. These data underpin the nature of the germline, which functions through cell cycle progression and differentiation. It also shows the necessity of the proteasome in germline development. In addition, as expected, the expression of genes encoding MSPs and sperm specific proteins was highly enriched in the germline.

To validate the gene expression changes observed between TES and VAS tissues, we selected 18 genes (Daf-21, Cul-1, Skr-1, Ubc-7, Rbx-1, Rpn-1, Pas-4, Pbs-2, Let-70, Eel-1, Kin-19, Sel-12, Paa-1 Gsk-3, Cdc-42, Smo1, Exos-7 and Pri-1) having significantly higher levels of expression in TES than in VAS for semi-quantitative RT-PCR analysis. These genes are involved in processes including, protein processing, ubiquitin-proteasome pathways, Wnt signaling, cell division and nucleic acid metabolism. The results (Additional file 6) showed that the expression in the majority of these genes is either down-regulated or absent in VAS as compared with TES.

\section{Comparison with C. elegans microarray and RNAi screening datasets}

Germline development in C. elegans has been extensively studied http://www.wormbook.org/toc_germline. html. A. suum and C. elegans belongs to Clade III and $\mathrm{V}$, respectively, in the phylum Nematoda, and it is 


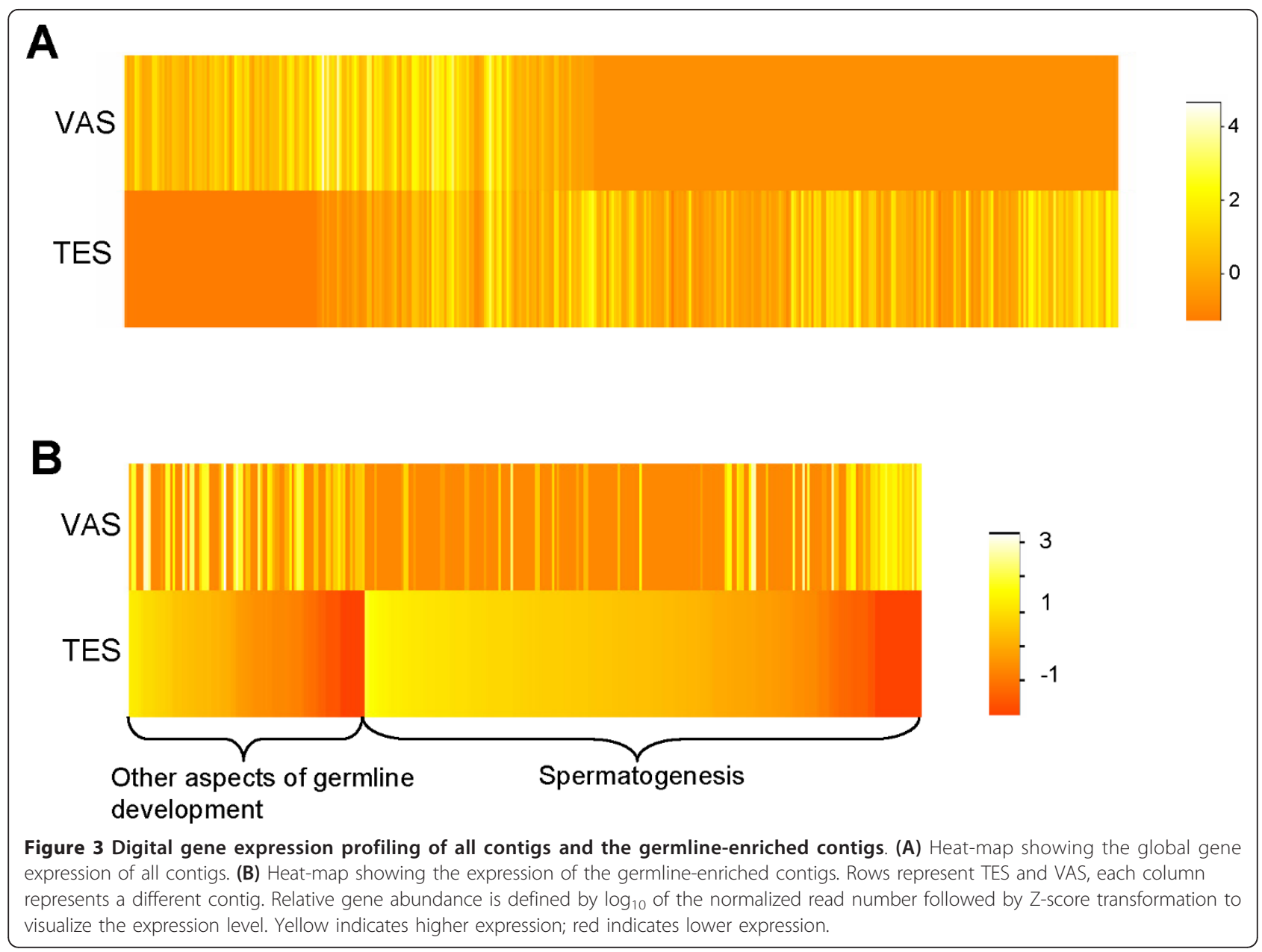

estimated that these two clades have an evolutionary divergence of 350 million years [20]. To identify the conserved genes regulating gonad development, we compared the A. suum gonad transcriptome with two $C$. elegans datasets acquired by microarray and genomewide RNAi analyses.

Using microarray technology, Reinke et al. identified 1,092 and 340 genes that have enriched expression in $C$. elegans adult male germline and soma, respectively (14 of these genes are pseudogenes or are no longer available in WormBase) [21]. BLAST analysis showed that 532 (49.1\%) of the C. elegans germline-enriched genes and 139 (32.8\%) of the soma-enriched genes have homologues in $A$. suum TES and VAS tissues, respectively (Additional file 7). The corresponding A. suum germline-enriched genes include 259 contigs and 37 singletons and the expression profiling of the 259 contigs is illustrated in Figure 3B. 165 genes of these contigs have over 10-fold higher levels of expression in TES than in VAS, and thus might represent conserved genes controlling germline development in different nematode species (Additional file 8). Among them, 137 genes (83\%) are spermatogenesis-enriched and the rest are involved in other aspects of germline development (e.g., mitotic proliferation). Substantial numbers of serine/threonine kinases and phosphatases (KPs), as well as tyrosine KPs were identified, suggesting pivotal roles of phosphorylation during spermatogenesis. It should be noted that the genes encoding KPs are over-represented among the sperm-enriched genes in C. elegans [22].

When comparing the TES and VAS datasets, we noticed an enriched expression of genes encoding KPs in TES tissues. There are 242 contigs in the TES dataset encoding KPs that have levels of expression 10-fold higher than those in VAS; in contrast, there are only 17 contigs encoding KPs with VAS/TES expression ratios above 10 (Additional file 9). The abundance of genes encoding KPs in TES tissues prompted us to examine the level of phosphorylation in total protein extracts from TES and VAS. Western blot analysis (Figure 4) showed that a $\sim 45 \mathrm{KD}$ protein (marked by arrowhead) in testis and sperm is associated with strong tyrosine phosphorylation (pY). The comparable amount of MSPs (marked by asterisk) in TES and sperm extracts (S100) 

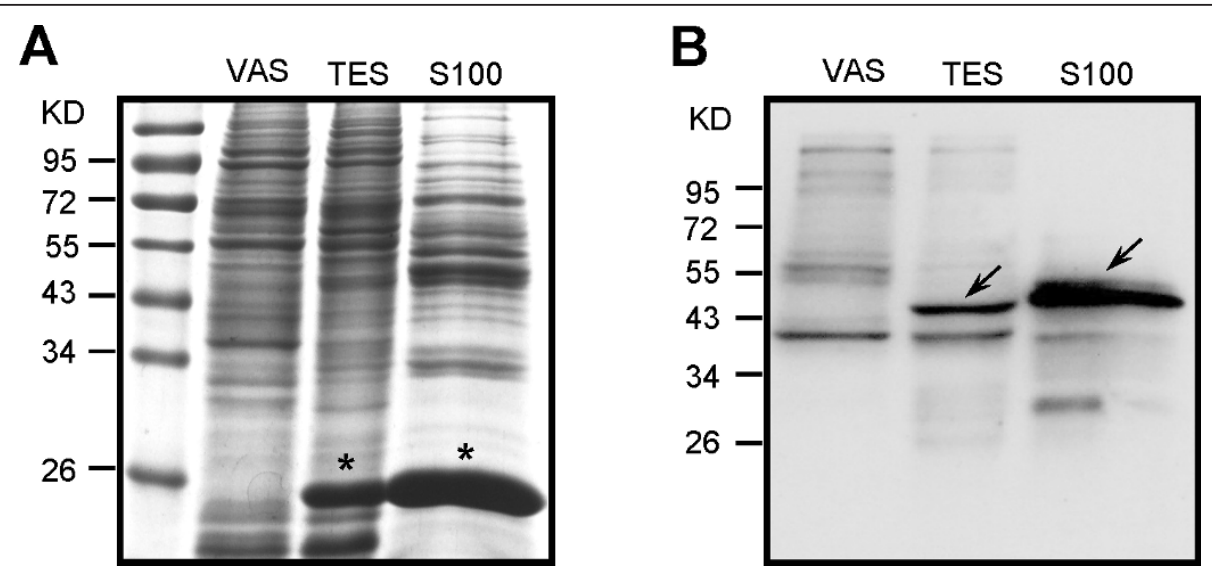

Figure 4 Western blot analysis of tyrosine phosphorylation ( $p Y$ ) in protein extracts of VAS, TES and sperm. (A) SDS-PAGE of total protein extracts of VAS, TES and sperm. Asterisks point to the MSPs that exist exclusively in sperm. (B) Western blot analysis of VAS, TES and sperm protein extracts probed with anti-pY. Arrows indicate a $\sim 45 \mathrm{KD}$ protein that is abundant in sperm extracts (S100).

indicates that this $\sim 45 \mathrm{KD}$ protein is abundant in sperm. In all soluble proteins of the sperm, this protein has the highest pY level suggesting that it may have essential functions during spermatogenesis or spermiogenesis. In contrast, the phosphorylated form of this protein was not detected in the vas deferens.

Through a genome-wide RNAi screen, Kalis et al. identified 207 genes which are essential for gonadogenesis in C. elegans (3 genes are no longer available in WormBase) [23]. BLAST analysis showed that 148 (71.5\%) of these genes have homologues represented in the A. suum gonad transcriptome (Additional file 10). This corresponded to $140 \mathrm{~A}$. suum genes and gene ontology (GO) analysis showed most of them regulate reproduction, embryo development, growth, locomotion and development of anatomical structures, as shown in Figure 5.

\section{Categorization of RNAi phenotypes of $A$. suum gonadal genes}

RNA interference (RNAi) was first discovered in $C$. elegans [24] and has since been widely used to suppress gene expression in a variety of organisms. Effective RNAi on A. suum larval development has been reported [25]. Therefore, we used the 11,968 genes that are orthologous between C. elegans and A. suum to query the $C$. elegans RNAi phenotype database. RNAi phenotypes associated with 2,681 genes were identified (Additional file 11). The main RNAi phenotypes are presented in Figure 6. These data show that a large portion of these genes are associated with embryonic lethality, slow growth, larval arrest, sterility and locomotion defects. This is in line with the functional classifications of the A. suum gonadal genes shown in Figure 5.

\section{Discussion and Conclusions}

Nematodes are one of the most diverse phyla and they make up approximately $80 \%$ of all individual animals on earth $[3,26]$. As the most prevalent nematode parasite in pigs, A. suum causes massive losses to the swine industry worldwide. The 959 Nematode Genomes Project has included this species for whole-genome sequencing and a draft genome and transcriptome of $A$. suum has just become available. In this study, we adopted 454 sequencing technology to determine the transcriptome of the A. suum gonad so as to facilitate further studies of this organism.

A total of 0.4 billion bp were obtained by 454 sequencing, which were assembled into 25 million bp, which is equivalent to the C. elegans exome (26 million bp). $86 \%$ of the high-quality reads were assembled into longer contigs, suggesting that these sequencing data had a high coverage. We annotated half of the contigs and $20 \%$ of the singletons; a large fraction of sequences have not been functionally assigned. The unannotated 454 ESTs may contain precursor non-coding RNAs (e.g., pre-miRNA, pre-snoRNAs) as well as the polyadenylated ncRNA classes; for example, over $13 \%$ and $26 \%$ of fulllength cDNAs in mice and human, respectively, are proposed to be polyadenylated mRNA-like ncRNAs [27-29]. The germline (TES) encompasses more gene models than the soma (VAS). Metabolic pathway mapping analysis also showed that TES and VAS datasets have distinct groups of genes involved in their respective metabolic processes.

To investigate germline-soma distinctions, we compared the digital transcriptomes of TES and VAS, and identified numerous TES-specific pathways, including DNA replication and proteasome and ubiquitinmediated proteolysis pathways. These pathways might 


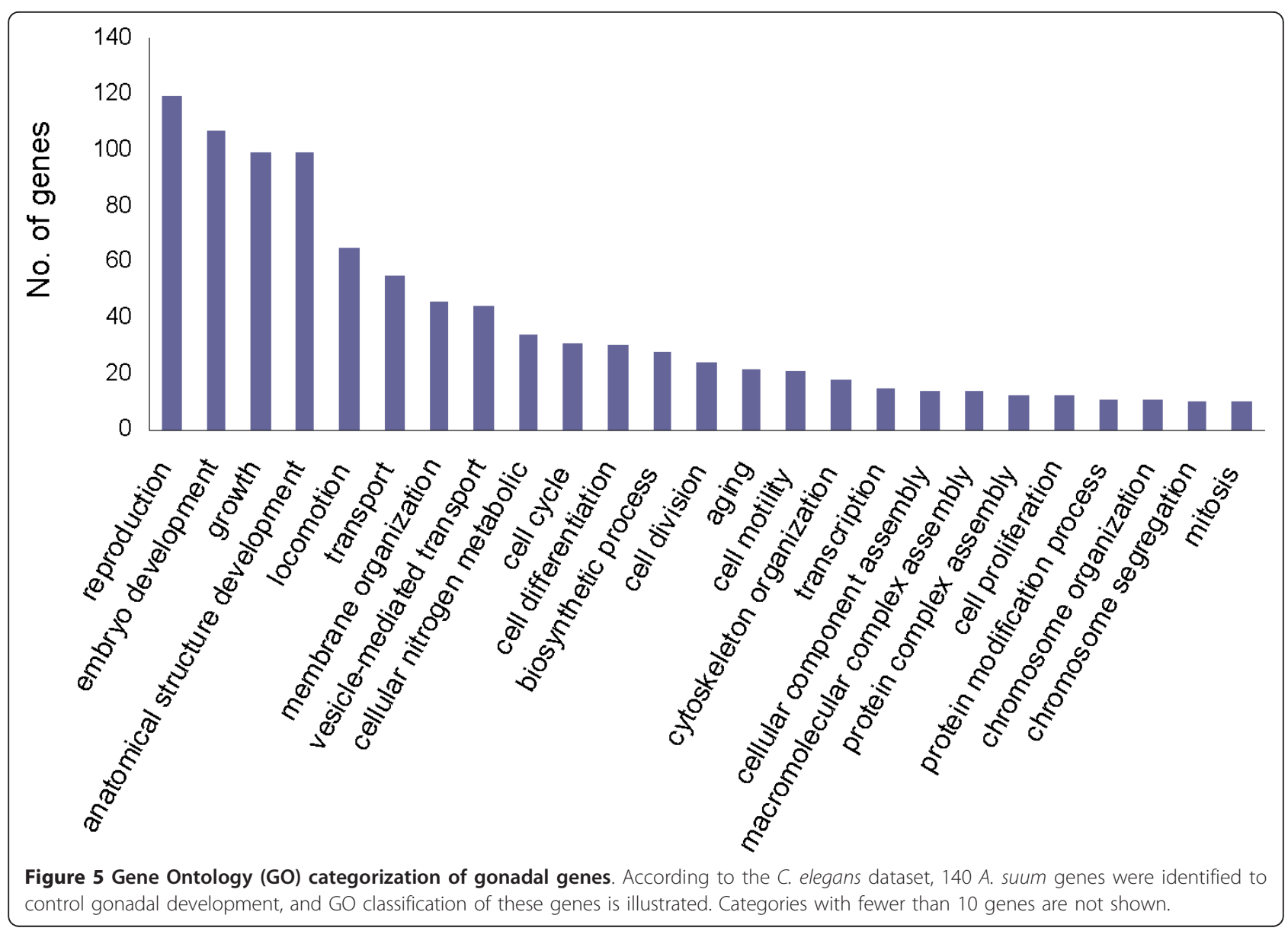

be required to regulate germline proliferation and differentiation. The proteasome has been documented to regulate the balance between cell proliferation and meiotic entry in C. elegans [30]. The genes encoding MSPs, sperm specific proteins and pyruvate dehydrogenase were the most highly expressed genes in the A. suum germline. MSP comprises $10-15 \%$ of the total proteins in nematode sperm [31] and sperm motility is driven by the regulated assembly and disassembly of MSP $[7,8,32,33]$. Hence, the high levels of expression of MSP genes were expected. With regard to pyruvate dehydrogenase, we speculate it might promote the tricarboxylic acid (TCA) cycle to supply energy for germ cell development.

We have a particular interest in A. suum spermatogenesis. Based on the C. elegans microarray data, $165 \mathrm{~A}$. suum genes (83\% are spermatogenesis-enriched) were identified as conserved genes controlling germline development. The most abundant proteins involved in $A$. suum spermatogenesis consist of sperm specific proteins, PDZ domain proteins, tyrosine kinases and phosphatases (KPs), and serine/threonine KPs. Identification of the genes encoding KPs in this analysis underpins the essential role of phosphorylation in the regulation of spermatogenesis. As the most common posttranslational modification, phosphorylation has been established to link to sperm function in a variety of species. In mammalians, the processes regulated by phosphorylation include capacitation, hyperactivated motility, zona pellucida binding, acrosome reaction and sperm-oocyte binding and fusion [34-36]. In C. elegans, the genes encoding KPs are over-represented among the spermenriched genes [22]. Clues to the phosphorylation signaling pathway that controls MSP-based cell motility were also documented in A. suum. A phosphorylated membrane protein (named MPOP) recruits a soluble casein kinase 1 (named MPAK) to the inner leaflet of the plasma membrane to initiate sperm motility [37]; MPAK, in turn, phosphorylates a second cytosolic protein (named MFP2) to accelerate MSP assembly $[10,11]$. A putative PP2A homologue was shown to trigger the retraction of MSP cytoskeleton [12]. The newly identified KPs in this study may aid in determining the signaling pathways that operate during spermatogenesis in $A$. suum. We provide evidence that a $\sim 45 \mathrm{KD}$ protein in sperm is associated with strong tyrosine phosphorylation 


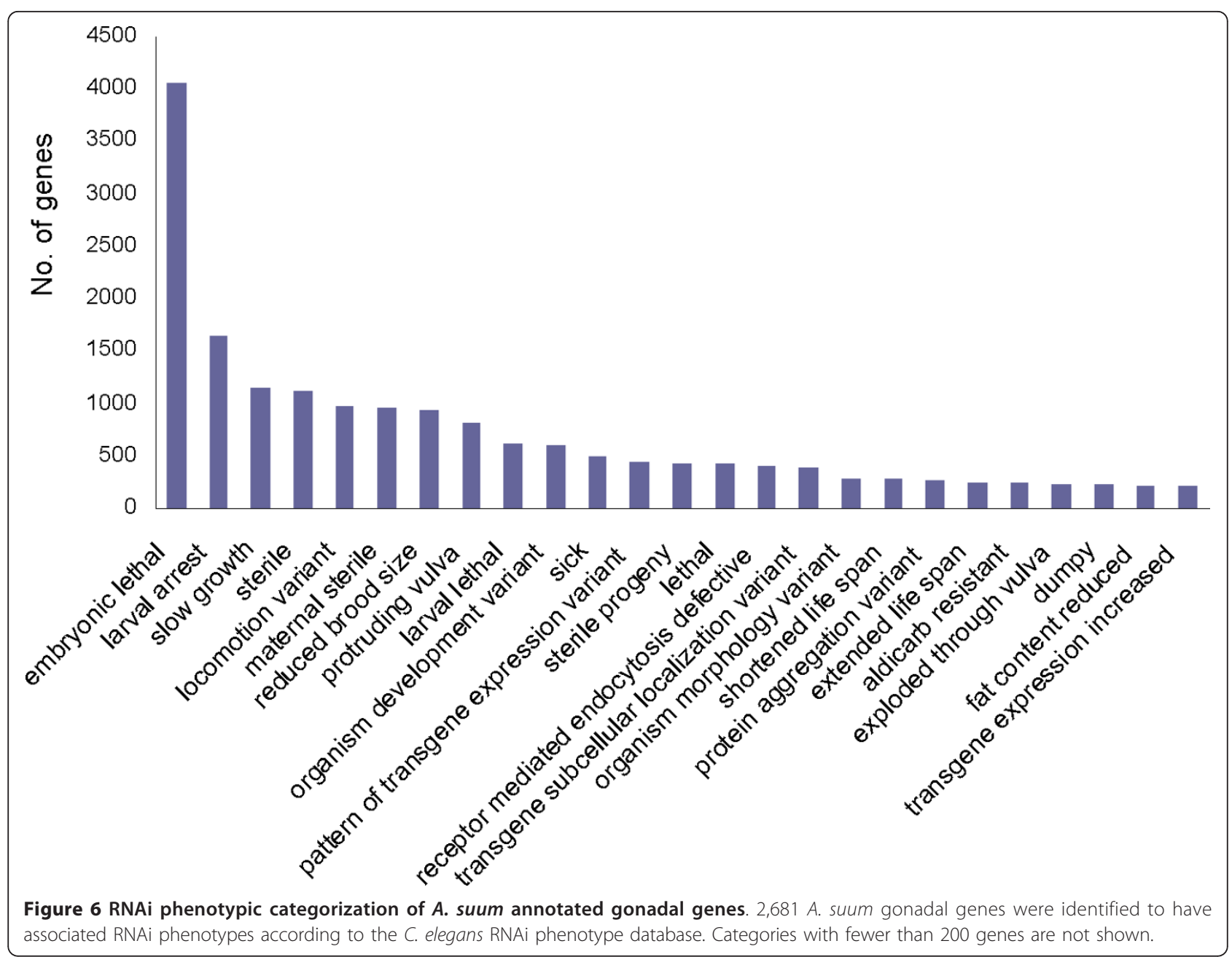

(pY). Because tyrosine phosphorylation and dephosphorylation act as a molecular switch to regulate MSP assembly [33], we propose this $\sim 45 \mathrm{KD}$ protein may be involved in MSP-based sperm motility. Immunolabeling of pY on the leading edge of spermatozoa has also been observed (Zhao Y. and Miao L., unpublished observations), which reinforces the notion that phosphorylation plays a role in A. suum spermatogenesis/spermiogenesis.

Lastly, $C$. elegans genes that are homologous to genes represented in the $A$. suum gonad transcriptome were examined for associated RNAi phenotypes. Although the genes involved in spermatogenesis are possibly insensitive to RNAi [38], a variety of RNAi phenotypes, mostly embryonic lethality, arrested growth or sterility, were retrieved from the C. elegans database. This RNAi phenotypic categorization is in line with the functional classification of the gonad developmental genes, the majority of which control reproduction, embryo development and growth. Due to the growing concern of anthelminth resistance, RNAi provides a new means to combat parasitic nematodes.
RNAi has been successfully used to knock down target genes in a few parasitic nematode species, including $B$. malayi [39], H. glycines [40], G. pallida [40], O. volvulus [41], T. colubriformis [42] and notably, A. suum [25]. Recently, serine/threonine phosphatases have been recommended as targets for new nematicidal drugs [43]. Therefore, we anticipate that these A. suum gonad transcriptome sequencing data will provide opportunities to use RNAi as a novel anti-parasite agent for parasite control.

\section{Methods}

\section{Collection of $A$. suum gonad samples}

A. suum males were collected from the intestines of infected hogs at Zhongrui Pork Processors (Liucun, Beijing, China) and were stored in worm buffer (phosphate buffered saline containing $100 \mathrm{mM} \mathrm{NaHCO}, \mathrm{pH}$ 7.0, $37^{\circ} \mathrm{C}$ ). A. suum gonads were dissected into two parts: (1) testis and seminal vesicle; (2) glandular vas deferens. Dissected samples were immediately frozen in liquid nitrogen prior to storage at $-80^{\circ} \mathrm{C}$. 


\section{cDNA synthesis and 454 pyrosequencing}

Total RNAs from A. suum testis and vas deferens were prepared using TRIzol reagent (Invitrogen, Carlsbad, CA, USA) before removal of trace genomic DNA using DNAse I (Promega, Madison, WI, USA). Poly A ${ }^{+}$RNA was purified using an Oligotex Direct mRNA Kit (Qiagen, Hilden, Germany) followed by first and secondstrand cDNA synthesis using the Universal RiboClone cDNA Synthesis System (Promega) with a modification as follows. We designed a poly(T) adaptor with a BsgI site flanking the poly $(\mathrm{T})$ sequence (5'-CGTGTGCAGT (20) VN-3') for cDNA synthesis. The purified secondstrand cDNAs were digested by $B s g \mathrm{I}$ and recovered with a QIAquick PCR Purification Kit (Qiagen). Both doublestrand cDNA samples were subjected to 454 pyrosequencing using the GS FLX Titanium Kit.

\section{4 sequencing data analyses}

High-quality sequences ( $>99.5 \%$ accuracy on single base reads) were filtered to remove short ESTs (<50 bp) before assembly using Newbler (version 2.3). For assembly, the quality score threshold was set to 40 . All unique sequences containing both contigs and singletons were compared against the $C$. elegans confirmed protein database (derived from Wormbase) as well as the KEGG protein databases for all organisms http://www.genome. jp/kegg/download/ by BLASTX; the BLAST cutoff Evalue was set at $<1 \mathrm{e}^{-5}$. The $A$. suum gonad developmental genes were functionally assigned by GO Slimer http://amigo.geneontology.org/cgi-bin/amigo/slimmer? session_id. The $A$. suum genes were mapped onto metabolic pathways according to C. elegans pathways http:// www.genome.jp/kegg/download/. Total read numbers of TES and VAS datasets were normalized to equal levels, and the relative gene abundance was defined by $\log _{10}$ of the normalized read number. Heat-maps were generated using R software (version 2.12.0).

\section{Reverse Transcription PCR}

Total RNAs from A. suum testis and vas deferens were isolated before being reverse transcribed into cDNA using the SuperScript III First-Strand Synthesis System (Invitrogen). The cDNA products were diluted 10-fold for use as RT-PCR templates. PCR was performed using High Fidelity PCR SuperMix (TransGen, Beijing, China) under the following conditions: $94^{\circ} \mathrm{C}$ for $3 \mathrm{~min} ; 30$ cycles of $94^{\circ} \mathrm{C}$ for $30 \mathrm{~s}, 57^{\circ} \mathrm{C}$ for $30 \mathrm{~s}, 72^{\circ} \mathrm{C}$ for $1 \mathrm{~min} ; 72^{\circ} \mathrm{C}$ for 8 min. The genes $A c t-1$ and $e I F-4 A$ were used as controls.

\section{Preparation of TES and VAS protein extracts and of sperm extracts (S100)}

Fresh A. suum TES and VAS tissues were disrupted in HKB buffer $(50 \mathrm{mM}$ Hepes, $65 \mathrm{mM} \mathrm{KCl,} 10 \mathrm{mM}$
$\mathrm{NaHCO}_{3}, \mathrm{pH}$ 7.1) in a homogenizer. An equal volume of lysis buffer (100 mM Hepes, $300 \mathrm{mM} \mathrm{NaCl}, 2 \%$ Triton-100) was added and samples were then placed on ice for $30 \mathrm{~min}$ before centrifugation for $30 \mathrm{~min}$ at 20,000 rpm. Supernatants were then collected for Western blot analysis. To prepare sperm extracts (S100), frozen sperm were thawed on ice for $1 \mathrm{hr}$ and centrifuged for $10 \mathrm{~min}$ at $13,000 \mathrm{rpm}$. Supernatant was centrifuged at $100,000 \times \mathrm{g}$ for $1 \mathrm{hr}$ at $4^{\circ} \mathrm{C}$. Supernatant (S100) was further analyzed by Western blotting.

\section{SDS-PAGE and Western blotting}

SDS-PAGE and Western blotting were performed as previously described [12]. Western blots were probed with anti-phosphotyrosine primary antibody (Millipore, Billerica, MA, USA) at $0.2 \mu \mathrm{g} / \mathrm{mL}$, followed by peroxidase-conjugated secondary antibody, and developed with enhanced chemiluminescence (PerkinElmer, Waltham, MA, USA).

\section{Additional material}

\begin{abstract}
Additional file 1: Summary of gene assignments for the $A$. suum testis EST library in NEMBASE4 and for the $A$. suum gonad transcriptome. Sheet GenBank EST summarizes the A. suum testis EST library; Sheet Gonad 454 data summarizes the A. suum gonad transcriptome.
\end{abstract}

Additional file 2: Annotation of A. suum germline (TES) and soma (VAS) transcriptomes

Additional file 3: Metabolic pathway assignment of $A$. suum TES and VAS transcriptomes.

Additional file 4: Transcripts with relatively high expression levels in TES and VAS. Sheet TES summarizes transcripts having a 10-fold or higher level of expression in TES compared with VAS; Sheet VAS summarizes transcripts having a 10-fold or higher level of expression in VAS compared with TES.

Additional file 5: Gene/Protein association network for genes highly expressed in TES. The functions of the highlighted gene clusters are as follows: gray, proteasome pathway; yellow, ubiquitin pathway; green, nucleotide metabolism; pink, ribosome members; blue, MSP family.

Additional file 6: RT-PCR verification of highly expressed genes in TES. Genes Act-1 and elF-4A are used as controls. Gene codes are from C. elegans.

Additional file 7: Predicted germline and soma-enriched genes in $A$. suum. Datasets were acquired by comparison of the A. suum gonad transcriptome with published $C$. elegans microarray data.

Additional file 8: Summary of the $165 \mathrm{~A}$. suum candidate genes conserved in nematode germline development.

Additional file 9: Contigs encoding kinases and phosphatases that are highly expressed in TES and VAS tissues. Sheet TES summarizes the transcripts of KPs having a 10-fold or higher level of expression in TES compared with VAS; Sheet VAS summarizes the transcripts of KPS having a 10-fold or higher level of expression in VAS compared with TES Additional file 10: Genes predicted to control gonadogenesis in $A$. suum. Dataset was acquired by comparison of the A. suum gonad transcriptome with published C. elegans genes that regulate gonad development.

Additional file 11: RNAi phenotypic categorization of $A$. suum gonadal genes. Each $A$. suum sequence ID is in bracket. RNAi phenotype codes are from Wormbase. 


\section{Acknowledgements}

We thank Dr. Yanmei Zhao for suggestions on total protein extraction from TES and VAS tissues and we thank Fugang Chen for dissections of TES and VAS tissues. This research was supported by grants 2012CB94502, 31171337 and 30971648 (to LM) and 81130069 (to SC) from the Chinese government. LM is supported by the Chinese Academy of Sciences 100-talents program.

\section{Author details}

'Noncoding RNA laboratory, National Laboratory of Biomacromolecules, Institute of Biophysics, Chinese Academy of Sciences, Beijing 100101, China. ${ }^{2}$ Institute of Medicinal Plant Development, Chinese Academy of Medical Sciences, Beijing 100094, China. ${ }^{3}$ Linyi Chest Hospital, Linyi 276034, Shandong Province, China.

\section{Authors' contributions}

XM and LM conceived and designed the experiments. SC helped to conceive and coordinate the experiments. FM participated in A. suum sample collection. XM prepared ds CDNA samples and performed RT-PCR. CL conducted library construction and 454 pyrosequencing. XM and YS extracted total proteins from TES and VAS tissues and performed Western blot analyses. XM and YZ performed data analyses. XM and LM wrote the manuscript. All authors have read and approved the final manuscript.

Received: 11 May 2011 Accepted: 1 October 2011

Published: 1 October 2011

\section{References}

1. Stewart TB, Hale OM: Losses to Internal Parasites in Swine Production. Journal of Animal Science 1988, 66:1548-1554.

2. Wolstenholme AJ, Fairweather I, Prichard R, von Samson-Himmelstierna G Sangster NC: Drug resistance in veterinary helminths. Trends in Parasitology 2004, 20(10):469-476.

3. Parkinson J, Mitreva M, Whitton C, Thomson M, Daub J, Martin J, Schmid R, Hall N, Barrell B, Waterston RH, et al: A transcriptomic analysis of the phylum Nematoda. Nature Genetics 2004, 36(12):1259-1267.

4. Ranganathan S, Menon R, Gasser RB: Advanced in silico analysis of expressed sequence tag (EST) data for parasitic nematodes of major socio-economic importance-Fundamental insights toward biotechnological outcomes. Biotechnology Advances 2009, 27(4):439-448.

5. Cantacessi C, Jex AR, Hall RS, Young ND, Campbell BE, Joachim A, Nolan MJ, Abubucker S, Sternberg PW, Ranganathan S, et al: A practical, bioinformatic workflow system for large data sets generated by nextgeneration sequencing. Nucleic Acids Research 2010, 38(17).

6. Fitzgerald LA, Foor WE: Nematoda. In Reproductive Biology of Invertebrates: Progress in Male Gamete Edited by: Adiyodi KG, Adiyodi RG 1988, III.

7. Roberts TM, Stewart M: Acting like actin: The dynamics of the nematode major sperm protein (MSP) cytoskeleton indicate a push-pull mechanism for amoeboid cell motility. Journal of Cell Biology 2000, 149(1):7-12

8. Stewart M, Roberts TM: Cytoskeleton dynamics powers nematode sperm motility. Fibrous Proteins: Muscle and Molecular Motors 2005, 71:383-+.

9. LeClaire LL, Stewart M, Roberts TM: A 48 kDa integral membrane phosphoprotein orchestrates the cytoskeletal dynamics that generate amoeboid cell motility in Ascaris sperm. Journal of Cell Science 2003, 116(13):2655-2663.

10. Buttery SM, Ekman GC, Seavy M, Stewart M, Roberts TM: Dissection of the Ascaris sperm motility machinery identifies key proteins involved in major sperm protein-based amoeboid locomotion. Molecular Biology of the Cell 2003, 14(12):5082-5088

11. Yi KX, Buttery SM, Stewart M, Roberts TM: A Ser/Thr kinase required for membrane-associated assembly of the major sperm protein motility apparatus in the amoeboid sperm of Ascaris. Molecular Biology of the Cell 2007, 18(5):1816-1825.

12. Yi KX, Wang X, Emmett MR, Marshall AG, Stewart M, Roberts TM: Dephosphorylation of Major Sperm Protein (MSP) Fiber Protein 3 by Protein Phosphatase 2A during Cell Body Retraction in the MSP-based Amoeboid Motility of Ascaris Sperm. Molecular Biology of the Cell 2009, 20(14):3200-3208.

13. Moritz KB, Roth GE: Complexity of Germ-Line and Somatic DNA in Ascaris. Nature 1976, 259(5538):55-57.
14. Muller F, Tobler $\mathrm{H}$ : Chromatin diminution in the parasitic nematodes Ascaris suum and Parascaris univalens. International Journal for Parasitology 2000, 30(4):391-399.

15. Muller F, Wicky C, Spicher A, Tobler H: New Telomere Formation after Developmentally Regulated Chromosomal Breakage during the Process of Chromatin Diminution in Ascaris-Lumbricoides. Cell 1991, 67(4):815-822.

16. Spicher A, Etter A, Bernard V, Tobler H, Muller F: Extremely Stable Transcripts May Compensate for the Elimination of the Gene Fert- 1 from All Ascaris-Lumbricoides Somatic-Cells. Developmental Biology 1994, 164(1):72-86.

17. Jentsch $\mathrm{S}$, Tobler $\mathrm{H}$, Muller F: New telomere formation during the process of chromatin diminution in Ascaris suum. International Journal of Developmental Biology 2002, 46(1):143-148

18. Etter A, Bernard V, Kenzelmann M, Tobler H, Muller F: Ribosomal Heterogeneity from Chromatin Diminution in Ascaris-Lumbricoides. Science 1994, 265(5174):954-956.

19. Kubota $Y$, Kuroki R, Nishiwaki K: A fibulin-1 homolog interacts with an ADAM protease that controls cell migration in C-elegans. Current Biology 2004, 14(22):2011-2018

20. Vanfleteren JRVdPY, Blaxter ML, Tweedie SA, Trotman C, Lu L, Van Hauwaert ML, Moens L: Molecular genealogy of some nematode taxa as based on cytochrome $\mathrm{c}$ and globin amino acid sequences. Molecular Phylogenetics and Evolution 1994, 3:92-101.

21. Reinke V, Gil IS, Ward S, Kazmer K: Genome-wide germline-enriched and sex-biased expression profiles in Caenorhabditis elegans. Development 2004, 131(2):311-323.

22. Kim SK, Reinke V, Smith HE, Nance J, Wang J, Van Doren C, Begley R, Jones SJM, Davis EB, Scherer S, et al: A global profile of germline gene expression in C-elegans. Molecular Cell 2000, 6(3):605-616.

23. Kalis AK, Kroetz MB, Larson KM, Zarkower D: Functional Genomic Identification of Genes Required for Male Gonadal Differentiation in Caenorhabditis elegans. Genetics 2010, 185(2):523-+

24. Fire A, Xu SQ, Montgomery MK, Kostas SA, Driver SE, Mello CC: Potent and specific genetic interference by double-stranded RNA in Caenorhabditis elegans. Nature 1998, 391(6669):806-811.

25. Xu MJ, Chen N, Song HQ, Lin RQ, Huang CQ, Yuan ZG, Zhu XQ: RNAimediated silencing of a novel Ascaris suum gene expression in infective larvae. Parasitology Research 2010, 107(6):1499-1503.

26. Lorenzen S: Phylogenetic Systematics of Freeliving Nematodes London: The Ray Society; 1994.

27. Okazaki Y, Furuno M, Kasukawa T, Adachi J, Bono H, Kondo S, Nikaido I, Osato N, Saito R, Suzuki H, et al: Analysis of the mouse transcriptome based on functional annotation of 60,770 full-length cDNAs. Nature 2002, 420(6915):563-573.

28. Ota T, Suzuki $Y$, Nishikawa T, Otsuki T, Sugiyama T, Irie R, Wakamatsu A, Hayashi $\mathrm{K}$, Sato $H$, Nagai $\mathrm{K}$, et al: Complete sequencing and characterization of 21,243 full-length human cDNAs. Nature Genetics 2004, 36(1):40-45.

29. Numata K, Kanai A, Saito R, Kondo S, Adachi J, Wilming LG, Hume DA, Hayashizaki $Y$, Tomita M, Grp RG, et al: Identification of putative noncoding RNAs among the RIKEN mouse full-length cDNA collection. Genome Research 2003, 13(6B):1301-1306.

30. MacDonald LD, Knox A, Hansen D: Proteasomal Regulation of the Proliferation vs. Meiotic Entry Decision in the Caenorhabditis elegans Germ Line. Genetics 2008, 180(2):905-920.

31. Klass M, Dow B, Herndon M: Cell-Specific Transcriptional Regulation of the Major Sperm Protein in Caenorhabditis-Elegans. Developmental Biology 1982, 93(1):152-164.

32. Italiano JE, Roberts TM, Stewart M, Fontana CA: Reconstitution in vitro of the motile apparatus from the amoeboid sperm of Ascaris shows that filament assembly and bundling move membranes. Cell 1996, 84(1):105-114.

33. Miao L, Vanderlinde $O$, Stewart M, Roberts TM: Retraction in amoeboid cell motility powered by cytoskeletal dynamics. Science 2003, 302(5649):1405-1407.

34. Tapia JA, Gonzalez-Fernandez L, Ortega-Ferrusola C, Macias-Garcia B, Salido GM, Pena FJ: Identification of Protein Tyrosine Phosphatases and Dual-Specificity Phosphatases in Mammalian Spermatozoa and Their Role in Sperm Motility and Protein Tyrosine Phosphorylation. Biology of Reproduction 2009, 80(6):1239-1252. 
35. Urner F, Sakkas D: Protein phosphorylation in mammalian spermatozoa. Reproduction 2003, 125(1):17-26.

36. Darszon A, Visconti PE, Krapf D, de la Vega-Beltran JL, Acevedo JJ: Ion channels, phosphorylation and mammalian sperm capacitation. Asian Journal of Andrology 2011, 13(3):395-405.

37. LeClaire LL, Stewart M, Roberts TM: A 48 kDa integral membrane phosphoprotein directs major sperm protein polymerization to the leading edge of crawling sperm from Ascaris. Molecular Biology of the Cell 2000, 11:179a-179a.

38. Chu DS, Liu HB, Nix P, Wu TF, Ralston EJ, Yates JR, Meyer BJ: Sperm chromatin proteomics identifies evolutionarily conserved fertility factors. Nature 2006, 443(7107):101-105.

39. Aboobaker AA, Blaxter ML: Use of RNA interference to investigate gene function in the human filarial nematode parasite Brugia malayi. Molecular and Biochemical Parasitology 2003, 129(1):41-51.

40. Urwin PE, Lilley CJ, Atkinson HJ: Ingestion of double-stranded RNA by preparasitic juvenile cyst nematodes leads to RNA interference. Molecular Plant-Microbe Interactions 2002, 15(8):747-752.

41. Lustigmana S, Zhang J, Liu J, Oksov Y, Hashmi S: RNA interference targeting cathepsin $L$ and $Z$-like cysteine proteases of Onchocerca volvulus confirmed their essential function during L3 molting. Molecular and Biochemical Parasitology 2004, 138(2):165-170.

42. Issa ZGW, Stasiuk S, Shoemaker CB: Development of methods for RNA interference in the sheep gastrointestinal parasite, Trichostrongylus colubriformis. International Journal for Parasitology 2005, 35(9):935-940.

43. Campbell BE, Hofmann A, McCluskey A, Gasser RB: Serine/threonine phosphatases in socioeconomically important parasitic nematodesProspects as novel drug targets? Biotechnology Advances 2011, 29(1):28-39.

doi:10.1186/1471-2164-12-481

Cite this article as: Ma et al:: Comparative transcriptome sequencing of germline and somatic tissues of the Ascaris suum gonad. BMC Genomics 2011 12:481.

\section{Submit your next manuscript to BioMed Central and take full advantage of:}

- Convenient online submission

- Thorough peer review

- No space constraints or color figure charges

- Immediate publication on acceptance

- Inclusion in PubMed, CAS, Scopus and Google Scholar

- Research which is freely available for redistribution

Submit your manuscript at www.biomedcentral.com/submit 